\title{
Oxygen saturation, red blood cell tissue fraction and speed resolved perfusion - A new optical method for microcirculatory assessment
}

Hanna Jonasson, Ingemar Fredriksson, Anders Pettersson, Marcus Larsson and Tomas

Strömberg

Linköping University Post Print

\section{Tweet}

N.B.: When citing this work, cite the original article.

Original Publication:

Hanna Jonasson, Ingemar Fredriksson, Anders Pettersson, Marcus Larsson and Tomas Strömberg, Oxygen saturation, red blood cell tissue fraction and speed resolved perfusion A new optical method for microcirculatory assessment, 2015, Microvascular Research, (102), 70-77.

http://dx.doi.org/10.1016/j.mvr.2015.08.006

Copyright: Elsevier

http://www.elsevier.com/

Postprint available at: Linköping University Electronic Press

http://urn.kb.se/resolve?urn=urn:nbn:se:liu:diva-121774 


\section{Oxygen saturation, red blood cell tissue fraction and speed resolved perfusion during local heating and occlusion provocations - A new optical method for microcirculatory assessment}

Hanna Jonasson ${ }^{\mathrm{a}, *}$, Ingemar Fredriksson ${ }^{\mathrm{a}, \mathrm{b}}$, Anders Pettersson ${ }^{\mathrm{b}}$, Marcus Larsson ${ }^{\mathrm{a}}$ and Tomas Strömberg

a Department of Biomedical Engineering, Linköping University, 58185 Linköping, Sweden.

${ }^{\text {b }}$ Perimed AB, Datavägen 9A, 17543 Järfälla, Stockholm, Sweden

* Corresponding author at: Department of Biomedical Engineering, Linköping University, 58185 Linköping, Sweden. Email address: hanna.jonasson@liu.se

\section{Abstract}

We have developed a new fiber-optic system that combines diffuse reflectance spectroscopy (DRS) and laser Doppler Flowmetry (LDF) for a multi-modal assessment of the microcirculation. Quantitative data is achieved with an inverse Monte Carlo algorithm based on an individually adaptive skin model. The output parameters are calculated from the model and given in absolute units: hemoglobin oxygen saturation (\%), red blood cell (RBC) tissue fraction (\%), and the speed resolved RBC perfusion separated into three speed regions; 0-1 mm/s, $1-10 \mathrm{~mm} / \mathrm{s}$ and above $10 \mathrm{~mm} / \mathrm{s}(\% \mathrm{~mm} / \mathrm{s})$. The aim was to explore microcirculatory parameters using the new optical method, integrating DRS and LDF in a joint skin model, during local heating of the dorsal foot and venous and arterial occlusion of the forearm in 23 healthy subjects (age 20-28 years). There were differences in the three speed regions in regard to blood flow changes due to local heating, where perfusion for high speeds increased the most. There was also a high correlation between changes in oxygenation and changes in perfusion for higher speeds. Oxygen saturation at baseline was $44 \%$ on foot, increasing to $83 \%$ at plateau after heating. The larger increase in perfusion for higher speeds than for lower speeds together with the oxygenation increase during thermal provocation, shows a local thermoregulatory blood flow in presumably arteriolar dermal vessels. In conclusion, there are improved possibilities to assess microcirculation using integrated DRS and LDF in a joint skin model by enabling both oxygenation and speed resolved blood flow assessment simultaneously and in the same skin site. Output parameters in absolute units may also yield new insights about the microcirculatory system.

Key words: diffuse reflectance spectroscopy, laser Doppler flowmetry, microcirculation, skin blood flow, hemoglobin oxygen saturation. 


\section{Introduction}

Recent studies show that there is a relationship between small vessel function and the heart and large vessel function [1]. There is increasing knowledge of the potential therapeutic/clinical value of the microcirculation as a model of vascular dysfunction on a systemic level $[2,3]$. Assessing vascular function is very promising in that it reflects important vascular biology associated with disease burden and outcome, and response to interventions. For example, endothelial dysfunction in terms of vascular reactivity is shown to be an integral component of atherosclerotic vascular disease and that its presence is a risk factor for the development of clinical events [4-6].

Diffuse reflectance spectroscopy (DRS) and laser Doppler flowmetry (LDF) are two non-invasive methods to assess the microcirculation in skin. Hemoglobin oxygenation and concentration of red blood cells (RBCs) can be assessed with DRS by using white light in the visible to near-infrared range where hemoglobin has absorption spectra with distinct features. With light transport in tissue modeling, knowledge of absorption spectra for hemoglobin and calibration of DRS spectra, oxygenation and tissue fraction of red blood cells can be determined in absolute units [7-9]. Also by using a small sourcedetector distance, the sampling depth becomes small and mostly vessels from the microcirculation affects the determined values.

LDF measures perfusion (concentration of RBCs times their average speed) of the tissue by registering the frequency shift, i.e. Doppler shift, occurring when light is scattered by moving RBCs. Conventionally, a perfusion measure is obtained by calculating the first moment of the detected Doppler spectrum. However, due to optical properties and structure of the tissue, the perfusion measure is expressed in arbitrary units which can be seen as a limitation of the technique $[10,11]$ since it complicates between subjects and between sites comparison. One way to overcome this is by using a model-based approach together with Monte Carlo simulations of light propagation in the tissue to obtain perfusion in absolute units [1214]. This is possible by performing an absolute calibration of the power in the measured spectra (see Data preprocessing below) whereas the frequencies are measured in absolute unit per se. By modelling the light transport with knowledge of the tissue optical properties in general (given from DRS measurements) and the scattering properties of RBC in specific (a priori knowledge), this model-based approach gives measured values in absolute units. In addition to the absolute values given from this approach and maybe even more important, the perfusion can also be separated into different speed regions of interest [12], which has also been shown by another group [15].

To combine these two techniques into the same probe has been suggested by other groups [16,17]. However, these systems analyze data from the two techniques separately, using conventional signal processing. This results in an uncertainty on the origin of the signal and the measured volume for the two systems, which is likely to be different [16]. By combining the two techniques into one joint model strengthens especially the robustness of the perfusion estimates, and the fact that one model can describe measured spectra from two different techniques increases the credibility of the method. Furthermore, with advanced light transport modelling, the output from the method can be obtained in absolute units, which makes it more compatible with other larger vessel measurement methods such as ultrasound.

With this newly developed state-of-the-art method for a more comprehensive characterization of the microcirculation, together with the new insight of the potential of microcirculatory assessments as a window to systemic pathophysiological conditions, suggest that this method could be an additional tool in clinical practice.

The aim of this study was to explore microcirculatory parameters using a new optical tool integrating DRS and LDF in a joint skin model. Standard provocations for determining the status of the microcirculation in the skin were performed; local heating of the foot and venous and arterial occlusion of the upper forearm. The output parameters oxygen saturation $\left(\mathrm{SO}_{2}\right)$, red blood cell tissue fraction $\left(C_{R B C}\right)$ and speed resolved perfusion were quantified during these provocations in absolute units. 


\section{Material and methods}

Subjects

Participants were non-smoking men and women with Caucasian skin type I-IV [18]. Exclusion criteria included disease or prescribed medication affecting the circulatory system, diabetes mellitus or hypertension. The participants were asked to refrain from coffee for $2 \mathrm{~h}$ prior to the measurements. Written informed consent was obtained from all participants. The study was approved by the Regional Ethical Review Board in Linköping (D. no M83-09).

\section{Equipment}

The DRS and LDF spectra were recorded using an integrated fiber optic probe (diameter: $200 \mu \mathrm{m}$; numerical aperture: 0.37; material: fused silica) with two emitting optical fibers (white light: Avalight-HAL-S, Avantes BV, The Netherlands; laser light: $780 \mathrm{~nm}$, modified Periflux 5000, Perimed AB, Järfälla, Sweden) and eight detecting fibers. DRS spectra were collected by two detector fibers at source-detector separation of 0.4 and $1.2 \mathrm{~mm}$, respectively. Each detector fiber was connected to a separate channel in a multi-channel spectrometer (AvaSpec 2048-5-RM, Avantes BV, The Netherlands, grating: VB 600 lines $/ \mathrm{mm}$ ). The remaining six detector fibers were all placed $1.2 \mathrm{~mm}$ from the laser optical source fiber and connected to a single detector in a modified Periflux 5000 (Perimed AB, Järfälla, Sweden). The probe was placed in a thermostatic probe holder (PF 450, Perimed AB, Järfälla, Sweden) fixated to skin using double-adhesive tape, to ensure stable skin temperature and good tissue contact.

\section{Measurement Protocols}

Measurements were performed in a room with an ambient temperature of $24.6 \pm 0.7^{\circ} \mathrm{C}$ (mean \pm standard deviation). The volunteers were acclimatized to the room temperature for at least $15 \mathrm{~min}$ before the start of the protocols. During the measurements, the subjects were in a supine position with their arms and legs fully rested. Three different provocations were conducted: local heating of the foot and venous and arterial occlusion of the upper forearm. During the local heating of the skin the probe was placed on the dorsum of the foot (right or left foot chosen randomly). During the venous occlusion the probe was placed on the volar surface of the lower forearm (right or left chosen randomly). The probe was then moved to the other lower forearm for the arterial occlusion. Before attaching the probe, the skin was cleaned with 95\% ethanol.

All protocols were preceded by a baseline of $5 \mathrm{~min}$, where the thermostatic probe holder had a temperature of $32^{\circ} \mathrm{C}$. During the heat provocation the temperature of the probe holder was increased to $44^{\circ} \mathrm{C}$ and held at that temperature for $25 \mathrm{~min}$. For the two occlusion provocations, the probe holder was kept at $32^{\circ} \mathrm{C}$. A blood-pressure cuff at a pressure of $60 \mathrm{mmHg}$ was used to perform a venous occlusion of the arm for $5 \mathrm{~min}$, followed by a 5 minute reperfusion phase. For the arterial occlusion, the cuff was inflated to $200 \mathrm{mmHg}$ for $5 \mathrm{~min}$ followed by a 5 minute reperfusion phase.

Spatiotemporal variation in the measurement/ reproducibility was assessed at baseline on the foot and on the forearm. On the foot, this was done by performing additional measurements of one minute before the local heating at two randomly chosen sites, avoiding visible veins (resulting in three baseline measurements sites). On the forearm, the two baseline sites measured before the provocations were used.

Data preprocessing

A recording from a dark spectrum and an intensity reference was performed before and after each measurement. The intensity reference was used as a link to a white spectrum, recorded at a separate occasion, and also for assessing color drift in the spectrometer during the measurement.

DRS spectra were calibrated in three steps: first the dark spectrum was subtracted, second the white reference spectrum was used to normalize DRS spectra, and third the two channels were normalized with a relative calibration using the average 
intensity from a measurement where the detecting fibers at the face of the probe was evenly illuminated [19]. Only the relative intensity between the two DRS channels is considered in the data processing, not the absolute light intensity levels.

LDF calibration was performed by measuring in motility standard (Perimed AB) where all detected light can be assumed to be Doppler shifted. The LDF spectra were normalized with the total spectral power of that calibration measurement. In that way, the power in the measured LDF spectra can be directly compared to the power in the simulated LDF spectra [20]. The conventional perfusion measure was calculated as the sum of the frequency weighted LDF spectrum, i.e. the first order moment, and normalized to $250 \mathrm{PU}$ (perfusion units) for the measurement in motility standard. The conventional CMBC (concentration of moving blood cells) measure was calculated as the total power of the LDF spectrum normalized to $400 \mathrm{CU}$ (concentration units) for the measurement in motility spectrum.

\section{Calculating microcirculatory parameters}

An adaptive three layer skin model $[13,21]$, was used to analyze DRS and LDF spectra. Modelled DRS and LDF spectra were generated based on Monte Carlo simulations of the model. The model parameters were updated in an iterative non-linear optimization manner in order for the modelled spectra to fit measured spectra. This was first done for DRS spectra for all model parameters except perfusion parameters. In a second step, the remaining model parameters, i.e. the perfusion parameters, were updated for the modeled LDF spectra to fit the measured LDF spectra. The output parameters i.e. $\mathrm{SO}_{2}$ in percent, $C_{R B C}$ in percent and perfusion in percent RBCs times their speed in $\mathrm{mm} / \mathrm{s}$, separated into three speed regions (0-1 $\mathrm{mm} / \mathrm{s}, 1-10 \mathrm{~mm} / \mathrm{s}$ and above $10 \mathrm{~mm} / \mathrm{s}$ ) were then calculated in the sampling volume that was also achieved from the model. The mean hemoglobin concentration of red blood cells was assumed to be $345 \mathrm{~g} / \mathrm{I} \mathrm{RBC}$.

\section{Microcirculatory evaluation}

DRS and LDF spectra were averaged over $1 \mathrm{~s}$ before analyzed using the adaptive individualized model. Baseline values for all provocations were calculated as the median value over the first three minutes of each measurement. First peak (median over one minute) and maximal thermal response (plateau, median over minute 22-25 after heat onset) were calculated from the heat provocation. The response after arterial occlusion for the perfusion measures was calculated as peak value and time to peak from release of the cuff pressure. $\mathrm{SO}_{2}$ and $\mathrm{C}_{\mathrm{RBC}}$ at time for total perfusion peak, together with maximal values (referred to as peak values) were also calculated. The response during occlusion was calculated as median value over the last minute, $10 \mathrm{~s}$ before release of the cuff pressure. The after occlusion measure was calculated as the median over minute 4-5 after release. The venous occlusion measures were calculated similar to the arterial occlusion, except for no peak value.

\section{Statistics}

Data are presented as mean \pm standard deviation (SD). Since some of the parameters were not normally distributed, Wilcoxon signed rank test was used for pairwise comparisons. Correlation coefficients between changes in $\mathrm{SO}_{2}, \mathrm{C}_{\mathrm{RBC}}$, perfusion for all speed regions as well as total perfusion and conventional perfusion during local heating were analyzed using Pearson correlation. A $p$-value $<0.05$ was considered significant. Statistical analyses were performed using IBM SPSS Statistics v21.0 (IBM Corporation, New York, USA).

Reproducibility was expressed as within subject coefficient of variation (CV) [22]

Data exclusion

To assess spectrometer color drift during the measurements, the calibration recording from the intensity reference before the start of the protocol was compared to the one after. The ratio of normalized intensity (before/after) was evaluated and if the spectral difference between maximal and minimal ratio was $>20 \%$, the subject was excluded [21]. 
Output parameters obtained from spectra with poor fitting for the DRS spectra or the LDF spectra were not included in the median value calculations. The $\mathrm{SO}_{2}$ level was considered uncertain if the hemoglobin spectral area (HemArea) in the 506 to $614 \mathrm{~nm}$ region for the DRS spectrum at $1.2 \mathrm{~mm}$ distance (Figure 1) was too small (less than $4 \%$ of the total spectral area, Total Area). If that was the case for more than $20 \%$ of the spectra during the measurement, $\mathrm{SO}_{2}$ was not calculated. A small HemArea is generally the result of a low $C_{R B C}$.

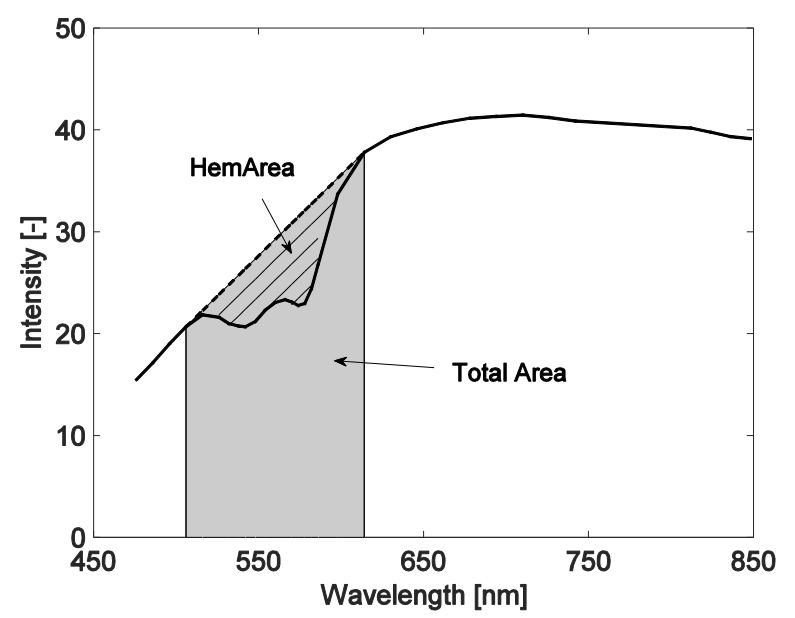

Figure 1. Diffuse reflectance spectra from source-detector distance $1.2 \mathrm{~mm}$. Definition of HemArea and Total Area for exclusion criteria.

\section{Results}

Measurements were performed on 23 subjects. Three subjects were excluded due to spectrometer color drift and three additional subjects were excluded due to missing before-and-after measurements on the intensity reference. One subject was excluded from the venous occlusion protocol due to the wrong pressure in the cuff. In total, measurements from 17 subjects were included in the local heating and arterial occlusion protocol, and 16 subjects during the venous occlusion protocol. The included subjects (10 male; 7 female) had a mean age $25 \pm 2$ years and BMI $23.8 \pm 3.0 \mathrm{~kg} / \mathrm{m}^{2}$.

The sampling depth, calculated as described in [13], was on average $0.67 \mathrm{~mm}$.

Reproducibility of baseline measurements

Variability was less than $40 \%$ for all parameters on the forearm as well as on the foot (Table 1). Highest variability (above $35 \%)$ was seen in perfusion for higher speeds on the foot. The variability was lowest for $\mathrm{SO}_{2}$ and low speed $(<1 \mathrm{~mm} / \mathrm{s})$ perfusion on both forearm and foot.

Table 1. Reproducibility at baseline on the forearm and on the foot.

\begin{tabular}{|l|l|l|}
\hline & \multicolumn{2}{|c|}{ CV [\%] } \\
\hline & Forearm & Foot \\
\hline $\mathrm{SO}_{2}$ & 10 & 14 \\
\hline $\mathrm{C}_{\mathrm{RBC}}$ & 17 & 24 \\
\hline Total perfusion & 22 & 26 \\
\hline Perfusion 0-1 mm/s & 10 & 17 \\
\hline Perfusion $1-10 \mathrm{~mm} / \mathrm{s}$ & 22 & 36 \\
\hline Perfusion $>10 \mathrm{~mm} / \mathrm{s}$ & 23 & 36 \\
\hline
\end{tabular}


Local heating

A representative example of the parameters during the local heating of the foot is presented in Figure 2. Total perfusion increased from $0.15 \% \mathrm{RBC} \times \mathrm{mm} / \mathrm{s}$ at baseline to $1.10 \% \mathrm{RBC} \times \mathrm{mm} / \mathrm{s}$ at first peak and $1.49 \% \mathrm{RBC} \times \mathrm{mm} / \mathrm{s}$ at plateau, while $\mathrm{SO}_{2}$ increased from $28 \%$ at baseline to $79 \%$ at first peak and $84 \%$ at plateau. There are small differences between the levels of the different speed regions during baseline, while upon heating the perfusion for high speeds ( $>10 \mathrm{~mm} / \mathrm{s}$ ) increased the most and perfusion for low speed $(0-1 \mathrm{~mm} / \mathrm{s})$ remained almost at baseline level.
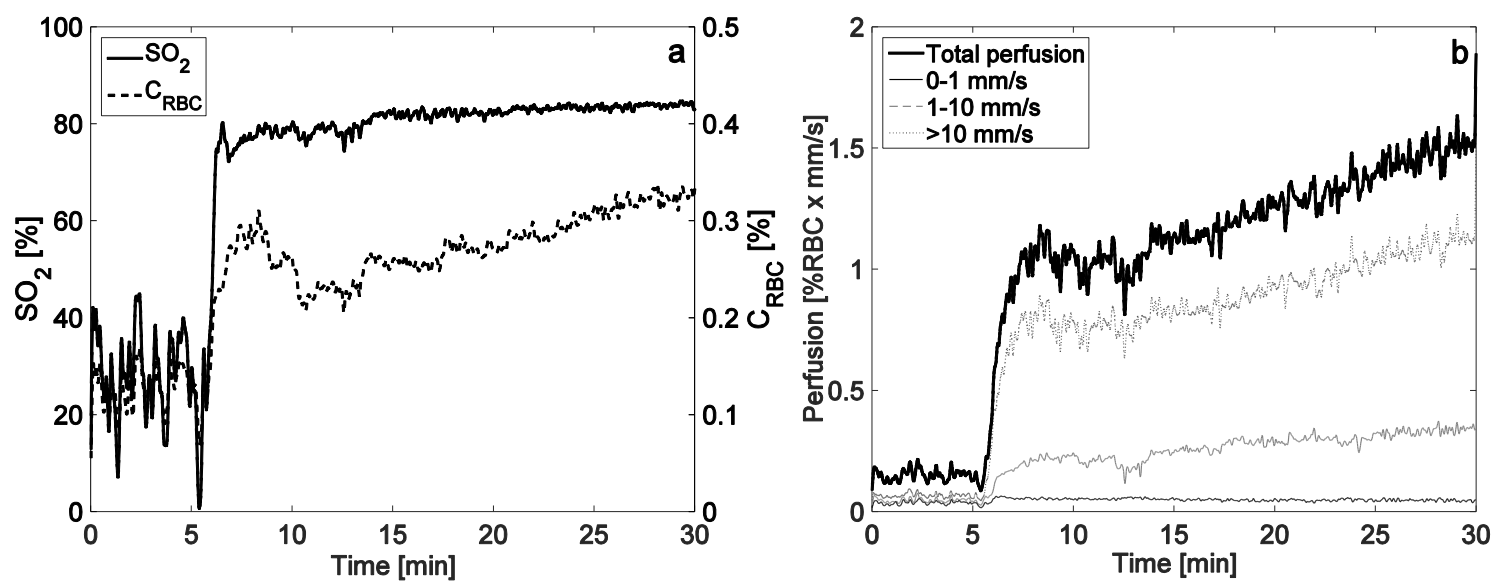

Figure 2. (a) Oxygen saturation $\left(\mathrm{SO}_{2}\right)$ and RBC tissue fraction $\left(C_{R B C}\right)$ during local heating of the foot. (b) Perfusion in percent RBC times speed divided into three speed regions $(0-1 \mathrm{~mm} / \mathrm{s}, 1-10 \mathrm{~mm} / \mathrm{s}$ and above $10 \mathrm{~mm} / \mathrm{s})$ together with total perfusion during local heating.

Mean values at baseline, first peak and plateau during local heating are presented in Table 2. $\mathrm{SO}_{2}$ for one subject during baseline was excluded due to small HemArea. The first peak, which mostly depends on local sensory nerves, was seen for all perfusion estimates but not for all subjects in the lowest speed region. It was not possible to distinguish a first peak for all subjects in $\mathrm{SO}_{2}$ (4 subjects), $\mathrm{C}_{\mathrm{RBC}}$ (3 subjects) or perfusion for the lowest speed region (4 subjects). There was a significant increase from baseline to first peak (in cases where first peak was identifiable) for all parameters $(p<0.01)$. The increase from baseline to plateau was also significant for all parameters ( $p<0.05$ or $p<0.01)$.

Table 2. Tissue model parameters during baseline and local heating of the foot; oxygen saturation $\left(\mathrm{SO}_{2}\right), \mathrm{RBC}$ tissue fraction ( $\mathrm{C}_{\mathrm{RBC}}$ ) and perfusion in percent $R B C$ times their speed (divided into speed regions $0-1 \mathrm{~mm} / \mathrm{s}, 1-10 \mathrm{~mm} / \mathrm{s}$, above $10 \mathrm{~mm} / \mathrm{s}$ and total perfusion). Conventional perfusion in perfusion units $(P U)$ and concentration of moving blood cells $(C M B C)$ in concentration units $(C U)$ are also given. $N=17$ if not other specified.

\begin{tabular}{|l|l|l|l|}
\hline & Baseline & First peak & Plateau \\
\hline $\mathrm{SO}_{2}[\%]$ & $44(14)^{1}$ & $79(7)^{4 \dagger}$ & $83(8)^{\dagger}$ \\
\hline $\mathrm{C}_{\mathrm{RBC}}[\%]$ & $0.17(0.088)$ & $0.25(0.099)^{3} \dagger$ & $0.30(0.11)^{\dagger}$ \\
\hline Total perfusion [\%RBC x mm/s] & $0.13(0.069)$ & $0.91(0.67)^{\dagger}$ & $1.26(0.77)^{\dagger}$ \\
\hline Perfusion 0-1 mm/s [\%RBC x mm/s] & $0.039(0.014)$ & $0.049(0.016)^{4} \dagger$ & $0.047(0.018)^{*}$ \\
\hline Perfusion 1-10 mm/s [\%RBC x mm/s] & $0.051(0.029)$ & $0.29(0.17)^{\dagger}$ & $0.37(0.17)^{\dagger}$ \\
\hline Perfusion >10 mm/s [\%RBC x mm/s] & $0.041(0.033)$ & $0.58(0.52)^{\dagger}$ & $0.84(0.64)^{\dagger}$ \\
\hline Conventional perfusion [PU] & $42(17)$ & $269(130)^{\dagger}$ & $356(129)^{\dagger}$ \\
\hline $\mathrm{CMBC}[\mathrm{CU}]$ & $287(31)$ & - & $318(28)^{\dagger}$ \\
\hline
\end{tabular}

Data expressed as mean (SD)

number of excluded values

Statistical comparison with baseline $* p<0.05 ; \dagger p<0.01$

The mean relative increase from baseline to plateau was $89 \%$ for $\mathrm{SO}_{2}, 76 \%$ for $\mathrm{C}_{\mathrm{RBC}}, 869 \%$ for the total perfusion and $748 \%$ for conventional perfusion. The increase for the different speed regions where $21 \%, 625 \%$ and $1950 \%$ (low, medium and high speeds). The conventional LDF measure concentration of moving blood cells (CMBC) increased $11 \%$. 
There was positive correlation between absolute changes (plateau-baseline) in $\mathrm{SO}_{2}$ and relative changes (plateau/baseline1) in total perfusion ( $r=0.75, p=0.001$, Figure 3.a), all speed regions (low: $r=0.61, p=0.012$; medium: $r=0.64, p=0.008$; high: $r=0.64, p=0.008)$ and conventional perfusion $(r=0.71, p=0.002)$. There were no correlation between absolute changes in $C_{R B C}$ and absolute changes in $\mathrm{SO}_{2}$, relative changes in total perfusion, perfusion for speed region $1-10 \mathrm{~mm} / \mathrm{s}$ or perfusion above $10 \mathrm{~mm} / \mathrm{s}$. However, there was a positive correlation between absolute changes in $\mathrm{C}_{\mathrm{RBC}}$ and relative changes in perfusion for the lowest speed region $(r=0.59, p=0.013)$. The relative changes in total perfusion were strongly correlated to those in conventional perfusion ( $r=0.92, p=0.000$, Figure $3 . b)$.
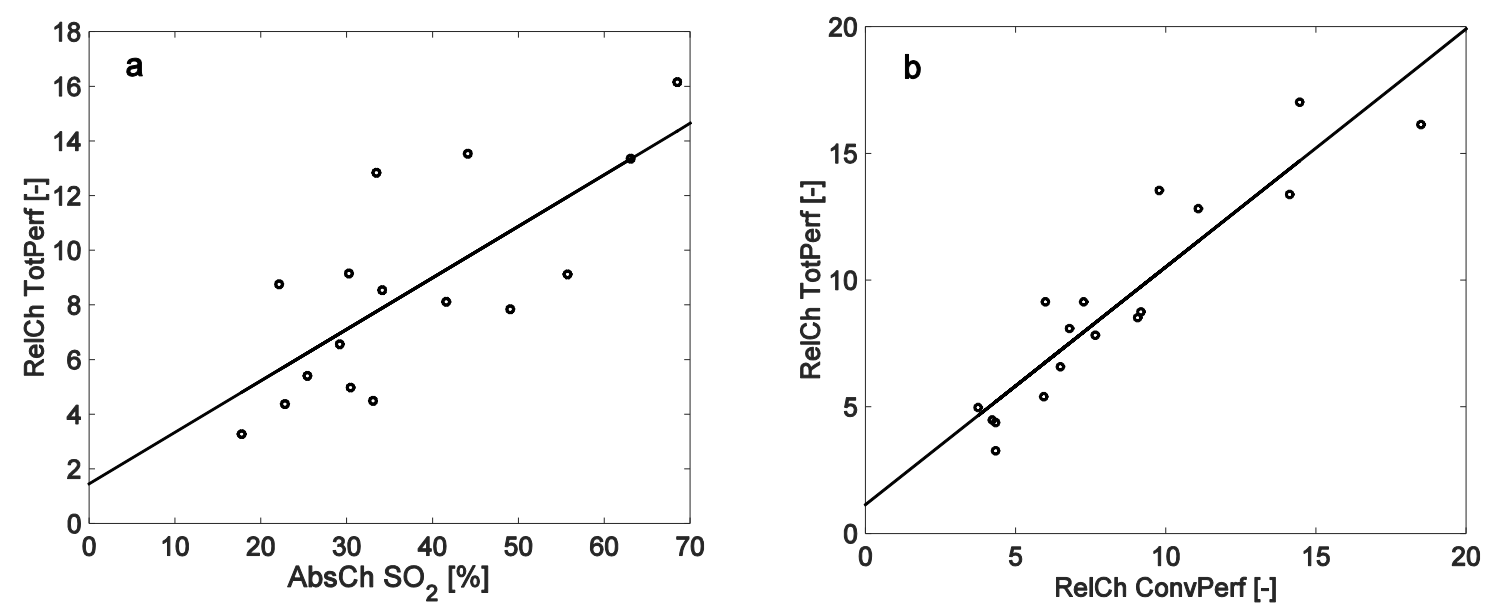

Figure 3. (a) Relative changes in total perfusion (RelCh TotPerf) and absolute changes in oxygen saturation ( $\mathrm{AbsCh} \mathrm{SO}_{2}$ in \%) (b) Relative changes in total perfusion (RelCh TotPerf) and conventional perfusion (RelCh ConvPerf).

Venous occlusion

A representative example of $\mathrm{SO}_{2}, \mathrm{C}_{\mathrm{RBC}}$ and perfusion parameters during venous occlusion is presented in Figure $4 . \mathrm{SO}_{2}$ was $58 \%$ at baseline and decreased slowly during the occlusion to $18 \%$. After release of the cuff, $\mathrm{SO}_{2}$ returned immediately to baseline values (52\%). RBC tissue fraction increased constantly during occlusion, from $0.12 \%$ baseline to $0.47 \%$ during occlusion and returned to $0.10 \%$ after release. Total perfusion was at baseline $0.14 \% \mathrm{RBC} \times \mathrm{mm} / \mathrm{s}$, decreased to $0.038 \% \mathrm{RBC}$ $x \mathrm{~mm} / \mathrm{s}$ during occlusion and returned to $0.083 \% R B C x \mathrm{~mm} / \mathrm{s} 4$ minutes after release. Perfusion for higher speeds $(>1 \mathrm{~mm} / \mathrm{s})$ were also lower than baseline values 4 minutes after release, while perfusion for the lowest speeds had returned to baseline values.
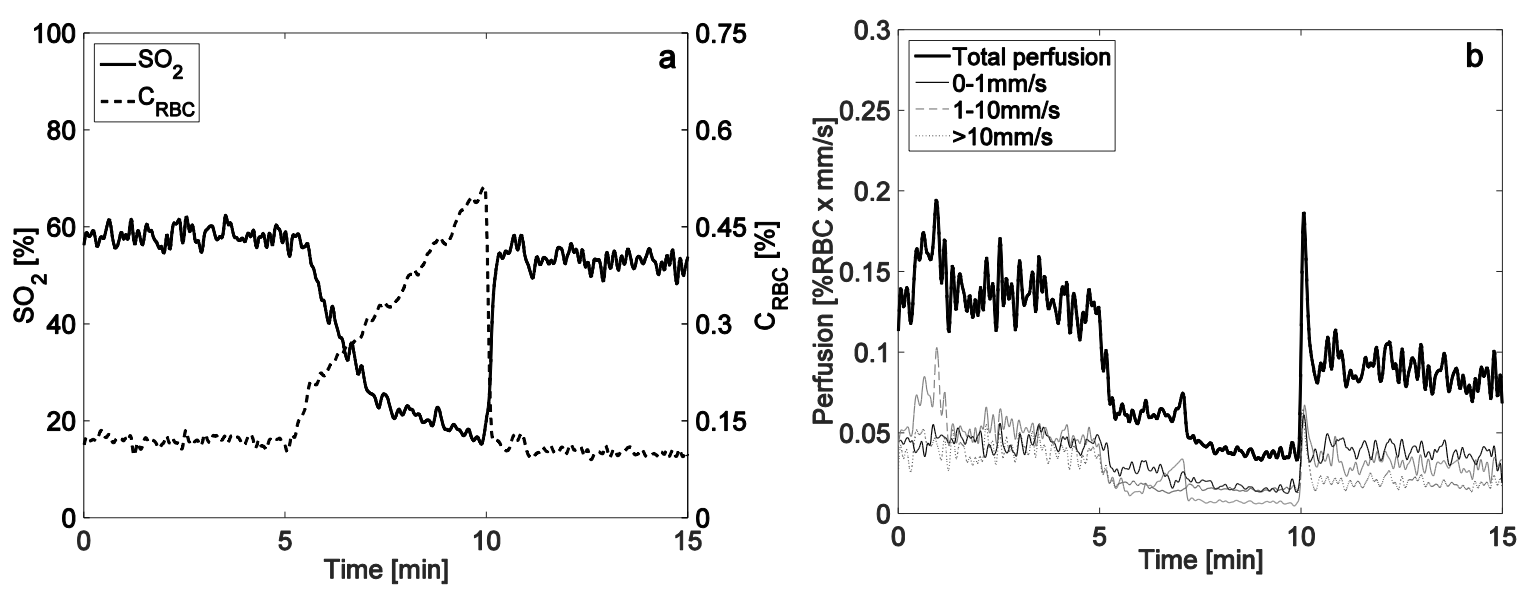
Figure 4. (a) Oxygen saturation $\left(\mathrm{SO}_{2}\right)$ and $\mathrm{RBC}$ tissue fraction $\left(\mathrm{C}_{\mathrm{RBC}}\right)$ during venous occlusion of the arm (b) Perfusion in percent RBC times speed divided into three speed regions $(0-1 \mathrm{~mm} / \mathrm{s}, 1-10 \mathrm{~mm} / \mathrm{s}$ and above $10 \mathrm{~mm} / \mathrm{s})$ together with total perfusion during venous occlusion.

Mean values at baseline, during and after venous occlusion are presented in Table $3 . \mathrm{SO}_{2}$ for one subject during baseline and after occlusion was excluded due to small HemArea. $\mathrm{SO}_{2}$ was significantly lower than the baseline value during occlusion, while $\mathrm{C}_{\mathrm{RBC}}$ was significantly higher $(p<0.01)$. All perfusion parameters were significantly lower during occlusion, including conventional perfusion $(p<0.01)$. After the release of the cuff $\mathrm{SO}_{2}, \mathrm{C}_{\mathrm{RBC}}$ and perfusion for lower speeds returned to baseline values after four minutes, but the total perfusion, conventional perfusion and perfusion for higher speeds were still significantly lower than baseline $(p<0.05$ or $p<0.01)$.

Table 3. Tissue model parameters during baseline and venous occlusion of the arm; oxygen saturation $\left(\mathrm{SO}_{2}\right), \mathrm{RBC}$ tissue fraction ( $\mathrm{C}_{\mathrm{RBC}}$ ) and perfusion in percent RBC times their speed (divided into speed regions $0-1 \mathrm{~mm} / \mathrm{s}, 1-10 \mathrm{~mm} / \mathrm{s}$, above $10 \mathrm{~mm} / \mathrm{s}$ and total perfusion). Conventional perfusion in perfusion units (PU) and concentration of moving blood cells $(C M B C)$ in concentration units [CU] are also given. $N=16$ if not other specified.

\begin{tabular}{|l|l|l|l|}
\hline & Baseline & $\begin{array}{l}\text { During venous } \\
\text { occlusion }\end{array}$ & After occlusion \\
\hline $\mathrm{SO}_{2}[\%]$ & $49(12)^{1}$ & $16(5)^{\dagger}$ & $49(13)^{1}$ \\
\hline $\mathrm{C}_{\mathrm{RBC}}[\%]$ & $0.15(0.067)$ & $0.56(0.29)^{\dagger}$ & $0.15(0.073)$ \\
\hline Total perfusion [\%RBC x mm/s] & $0.14(0.094)$ & $0.048(0.027)^{\dagger}$ & $0.11(0.098)^{*}$ \\
\hline Perfusion 0-1 mm/s [\%RBC x mm/s] & $0.031(0.012)$ & $0.015(0.0077)^{\dagger}$ & $0.029(0.014)$ \\
\hline Perfusion 1-10 mm/s [\%RBC x mm/s] & $0.052(0.045)$ & $0.013(0.0093)^{\dagger}$ & $0.039(0.034)^{*}$ \\
\hline Perfusion >10 mm/s [\%RBC x mm/s] & $0.054(0.073)$ & $0.020(0.017)^{\dagger}$ & $0.042(0.088)^{\dagger}$ \\
\hline Conventional perfusion [PU] & $48(29)$ & $19(9)^{\dagger}$ & $38(24)^{*}$ \\
\hline CMBC [CU] & $279(31)$ & $277(27)$ & $278(31)$ \\
\hline
\end{tabular}

Data expressed as mean (SD)

nnumber of excluded values

Statistical comparison with baseline ${ }^{*} p<0.05 ;+p<0.01$

CMBC did not change during the venous occlusion. The RBC tissue fraction on the other hand had a mean increase of $273 \%$ during the occlusion. $\mathrm{SO}_{2}$ decreased $65 \%$, the total perfusion $66 \%$ and conventional perfusion $60 \%$. The different speed regions decreased $52 \%, 75 \%$ and $63 \%$ (low, medium and high speeds).

Arterial occlusion

A representative example of $\mathrm{SO}_{2}, \mathrm{C}_{\mathrm{RBC}}$ and perfusion measures during arterial occlusion is presented in Figure $5 . \mathrm{SO}_{2}$ was $42 \%$ at baseline and decreased during the occlusion to $-2 \%$. Maximum value at release was $90 \%$ and four minutes after release, $\mathrm{SO}_{2}$ was $65 \%$ which was higher than baseline. $\mathrm{RBC}$ tissue fraction increased during occlusion, from $0.16 \%$ baseline to a maximum of $0.32 \%$ during occlusion and returned slowly to $0.14 \%$ after release. Total perfusion was at baseline 0.089 $\% \mathrm{RBC} \times \mathrm{mm} / \mathrm{s}$, decreased to $0.044 \% \mathrm{RBC} \times \mathrm{mm} / \mathrm{s}$ during occlusion, peaked at $0.69 \% \mathrm{RBC} \times \mathrm{mm} / \mathrm{s}$ and returned to $0.12 \% \mathrm{RBC}$ $\mathrm{x} \mathrm{mm} / \mathrm{s} 4$ minutes after release. 

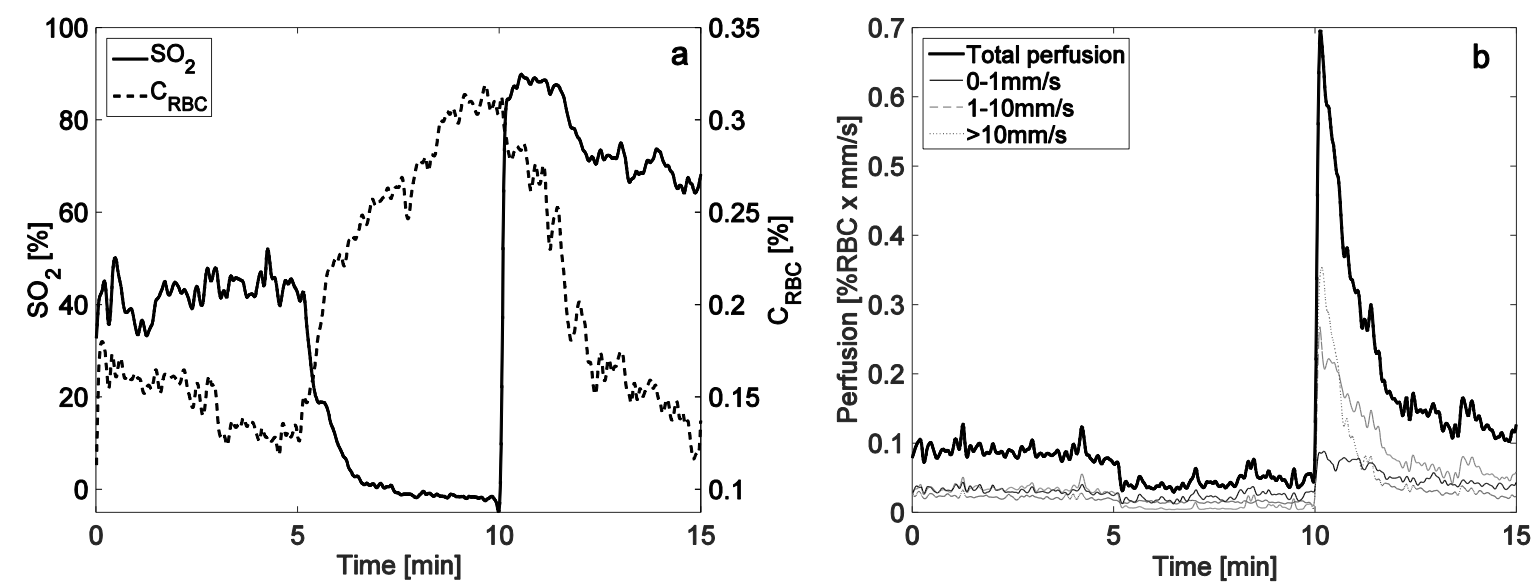

Figure 5. (a) Oxygen saturation $\left(\mathrm{SO}_{2}\right)$ and RBC tissue fraction $\left(C_{R B C}\right)$ during arterial occlusion of the arm (b) Perfusion in percent RBC times speed divided into three speed regions $(0-1 \mathrm{~mm} / \mathrm{s}, 1-10 \mathrm{~mm} / \mathrm{s}$ and above $10 \mathrm{~mm} / \mathrm{s})$ together with total perfusion during arterial occlusion.

Mean values at baseline, during occlusion, at peak and after arterial occlusion are presented in Table $4 . \mathrm{SO}_{2}$ for one subject during baseline, during occlusion and after occlusion was excluded due to small HemArea. It was not possible to distinguish a post occlusive peak for two subjects in the lowest speed region. All perfusion parameters and $\mathrm{SO}_{2}$ were significantly lower than baseline values during occlusion $(p<0.01)$, while $C_{\mathrm{RBC}}$ was significantly higher $(p<0.01)$. All parameters were significantly higher than baseline at peak $(p<0.01)$. Both $\mathrm{SO}_{2}$ and $\mathrm{C}_{\mathrm{RBC}}$ were at $91 \%$ of maximal value at the time for total perfusion peak. $\mathrm{SO}_{2}$ reaches maximal value after total perfusion peak for all subjects and $\mathrm{C}_{\mathrm{RBC}}$ reaches maximal value both before and after time for total perfusion peak. $\mathrm{SO}_{2}$ was still significantly higher than baseline value $4 \mathrm{~min}$ after the release of the cuff $(p<0.01)$. Note the difference between total perfusion and conventional perfusion after occlusion, where total perfusion was at baseline level while conventional perfusion was still significantly higher than baseline $(p<0.01)$.

Table 4. Tissue model parameters during baseline and arterial occlusion of the arm; oxygen saturation $\left(\mathrm{SO}_{2}\right), \mathrm{RBC}$ tissue fraction ( $\left.\mathrm{C}_{\mathrm{RBC}}\right)$ and perfusion in percent RBC times their speed (divided into speed regions $0-1 \mathrm{~mm} / \mathrm{s}, 1-10 \mathrm{~mm} / \mathrm{s}$, above $10 \mathrm{~mm} / \mathrm{s}$ and total perfusion). Conventional perfusion in perfusion units (PU) and concentration of moving blood cells $(C M B C)$ in concentration units $[C U]$ are also given. For $\mathrm{SO}_{2}$ and $C_{R B C}$ maximal values are referred to as peak values. $\mathrm{N}=17$ if not other specified.

\begin{tabular}{|l|l|l|l|l|}
\hline & Baseline & During occlusion & Peak & After occlusion \\
\hline $\mathrm{SO}_{2}[\%]$ & $47(13)^{1}$ & $-2(3)^{1 \dagger}$ & $84(6)^{\dagger}$ & $63(12)^{1 \dagger}$ \\
\hline $\mathrm{C}_{\mathrm{RBC}}[\%]$ & $0.16(0.051)$ & $0.28(0.12)^{\dagger}$ & $0.35(0.12)^{\dagger}$ & $0.15(0.043)$ \\
\hline $\begin{array}{l}\text { Total perfusion [\%RBC } \times \\
\mathrm{mm} / \mathrm{s}]\end{array}$ & $0.10(0.039)$ & $0.029(0.0088)^{\dagger}$ & $0.70(0.33)^{\dagger}$ & $0.11(0.058)$ \\
\hline $\begin{array}{l}\text { Perfusion 0-1 mm/s } \\
{[\% \mathrm{RBC} \times \mathrm{mm} / \mathrm{s}]}\end{array}$ & $0.031(0.013)$ & $0.012(0.0059)^{\dagger}$ & $0.070(0.023)^{2 \dagger}$ & $0.032(0.013)$ \\
\hline $\begin{array}{l}\text { Perfusion } 1-10 \mathrm{~mm} / \mathrm{s} \\
{[\% \mathrm{RBC} \times \mathrm{mm} / \mathrm{s}]}\end{array}$ & $0.042(0.019)$ & $0.0044(0.0023)^{\dagger}$ & $0.28(0.15)^{\dagger}$ & $0.051(0.031)$ \\
\hline $\begin{array}{l}\text { Perfusion }>10 \mathrm{~mm} / \mathrm{s} \\
{[\% \mathrm{RBC} \times \mathrm{mm} / \mathrm{s}]}\end{array}$ & $0.027(0.013)$ & $0.012(0.0033)^{\dagger}$ & $0.38(0.19)^{\dagger}$ & $0.031(0.023)$ \\
\hline $\begin{array}{l}\text { Conventional perfusion } \\
{[\mathrm{PU}]}\end{array}$ & $35(11)$ & $11(2)^{\dagger}$ & $215(62)^{\dagger}$ & $43(24)^{*}$ \\
\hline $\mathrm{CMBC}[\mathrm{CU}]$ & $279(29)$ & $226(39)^{\dagger}$ & - & $286(35)^{*}$ \\
\hline
\end{tabular}

Data expressed as mean (SD)

number of excluded values

Statistical comparison with baseline $* p<0.05 ;+p<0.01$

CMBC decreased $19 \%$ during occlusion while $\mathrm{C}_{\mathrm{RBC}}$ increased $75 \% . \mathrm{SO}_{2}$ decreased $104 \%$, total perfusion $71 \%$ and conventional perfusion $69 \%$. The different speed regions decreased $61 \%, 91 \%$ and $56 \%$ (low, medium and high speeds, respectively). 
Mean time to peak values are given in Table 5. Time to peak for perfusion for speeds $1-10 \mathrm{~mm} / \mathrm{s}$ was significant longer than time to peak for the total perfusion $(p<0.05)$.

Table 5. Time to peak for total perfusion, perfusion for speed regions $0-1 \mathrm{~mm} / \mathrm{s}, 1-10 \mathrm{~mm} / \mathrm{s}$, above $10 \mathrm{~mm} / \mathrm{s}$ and conventional perfusion. $\mathrm{N}=17$ if not other specified.

\begin{tabular}{|l|l|l|l|l|l|}
\hline & Total perfusion & $\begin{array}{l}\text { Perfusion 0-1 } \\
\mathbf{m m} / \mathbf{s}\end{array}$ & $\begin{array}{l}\text { Perfusion 1- } \\
\mathbf{1 0 ~} \mathbf{m m} / \mathbf{s}\end{array}$ & $\begin{array}{l}\text { Perfusion }>\mathbf{1 0} \\
\mathbf{m m} / \mathbf{s}\end{array}$ & $\begin{array}{l}\text { Conventional } \\
\text { perfusion }\end{array}$ \\
\hline $\begin{array}{l}\text { Time to peak } \\
{[\mathrm{s}]}\end{array}$ & $15(6)$ & $10(5)^{2}$ & $19(9)^{*}$ & $14(5)$ & $16(8)$ \\
\hline
\end{tabular}

Data expressed as mean (SD)

nnumber of excluded values

Statistical comparison with total perfusion $* p<0.05$

\section{Discussion}

We assessed microcirculatory parameters for healthy subjects using a new method, integrating diffuse reflectance spectroscopy and laser Doppler flowmetry in a combined skin model. The oxygen saturation, RBC tissue fraction and speed resolved perfusion, in absolute units, were evaluated during local heating of the foot and venous and arterial occlusion of the arm. Assessing these parameters simultaneously and in the same skin site enables studying the true interrelationship between the parameters. This real time analyze method may provide new insight into the microcirculation of the tissue and aid in a more comprehensive assessment of microvascular status.

Spatio-temporal variability at baseline/ reproducibility, was acceptable $(<35 \%$; [23]) on the forearm for all parameters and this indicates that the method has a better reproducibility compared to other studies regarding reproducibility of LDF on forearm [23]. When applying the method on the foot the variability was above $35 \%$ for perfusion for speeds above $1 \mathrm{~mm} / \mathrm{s}$. It is logical to assume that the spatial heterogeneity is higher for larger vessels since they are less densely distributed than smaller vessels. That could possible explain the larger $\mathrm{CV}$ for higher speeds.

$\mathrm{SO}_{2}$ during baseline, at peak after arterial occlusion and after heating were comparable to other studies [16, 17, 24], although the sampling volume differs between the studies. $\mathrm{SO}_{2}$ and $\mathrm{C}_{\mathrm{RBC}}$ on forearm at baseline and during occlusion were comparable to our previous findings [21], using DRS modeling. The higher $\mathrm{SO}_{2}$ peak after release in this study was probably due to the elevated cuff pressure from $30 \mathrm{mmHg}$ above systolic pressure to $200 \mathrm{mmHg}$, which was seen as a limitation in the previous study [21]. We have previously presented perfusion values in absolute units and in separate speed regions in healthy controls [25]. The achieved values are on a similar level, but they cannot be directly compared since the LDF part of the presented method differs in multiple ways from the previous LDF-only method. In the new integrated DRS and LDF method the sampling depth is different (different source-detector fiber separation and different way to weight layers in adapted model), a compensation for inhomogeneously distributed blood is implemented and the perfusion estimates benefit from model parameters obtained from the DRS system which improves the robustness. The conventional perfusion estimates in this study originates from a source-detector distance of $1.2 \mathrm{~mm}$ which is different from the mostly often used standard Perimed probe, and cannot therefore be compared to previously presented values.

The average sampling depth for the method, $0.67 \mathrm{~mm}$, is coherent with previous findings [26]. This sampling depth includes the dermal layers where capillaries, arterioles and venules are found. Larger vessels are normally associated with higher blood flow speeds than smaller vessels. Therefore, the speed resolved perfusion measures may aid in interpreting which vessels types that take part in the physiological response.

During local heating there was a difference in the different speed regions regarding the response. The increase from baseline to plateau was $869 \%$ for the total perfusion and $21 \%, 625 \%$ and $1950 \%$ (low, medium and high speeds, respectively) for the 
different speed regions. The higher relative change in perfusion for higher speeds is most likely due to the different physiology in the different vessel structures they represent. More smooth muscle cells are involved in larger vessels resulting in a larger vasodilation compared to smaller vessel structures which consist primarily of capillaries that have smaller vasoactive function. Well known mediators of smooth muscle cells are prostaglandins, nitric oxide and endotheliumderived hyperpolarizing factor, factors shown to be involved in thermal provocations as well [27]. The higher relative change in perfusion for higher speeds is also according to our previous findings in healthy controls [25]. The $\mathrm{SO}_{2}$ response supports the assumption of a non-metabolic flow, since there was a strong correlation between absolute changes in $\mathrm{SO}_{2}$ and relative changes in perfusion for higher speeds. The correlation between $\mathrm{SO}_{2}$ and the lowest speed region was weaker.

A major difference between $\mathrm{CMBC}$ and $\mathrm{C}_{\mathrm{RBC}}$ is that $\mathrm{CMBC}$ only involves red blood cells in motion and this causes differences in response between $\mathrm{CMBC}$ and $\mathrm{C}_{\mathrm{RBC}}$ during the provocations. In addition, for the fiber separation used in this study almost all detected photons that penetrates the non-perfused top epidermal layer will be Doppler shifted already at low levels of moving RBC. This result in a CMBC estimate that already at baseline is close to saturated with a low degree of dynamics. These differences are clearly displayed in the response to local heating were the $\mathrm{CMBC}$ only increased $11 \%$ while the $\mathrm{C}_{\mathrm{RBC}}$ increased $76 \%$. Similarly, during venous occlusion $C M B C$ remained practically non-affected of the occlusion while $C_{R B C}$ increased $273 \%$. During the arterial occlusion CMBC decreased $19 \%$ while $\mathrm{C}_{\mathrm{RBC}}$ increased $75 \%$.

It should be emphasized that the presented technique measure the fraction of RBC in the sampling volume, not the blood amount. The relationship between $C_{R B C}$ and blood tissue fraction is directly dependent on the (local) hematocrit. For normal hematocrit values of $40-50 \%$, the blood tissue fraction is thus slightly more than the double $C_{R B C}$. Note, however, that the hematocrit can vary within the vessel tree and can be significantly lower in the capillaries [28].

At release after arterial occlusion the $\mathrm{SO}_{2}$ peak was slower than the total perfusion since at the time for perfusion peak, $\mathrm{SO}_{2}$ was on average at $91 \%$ of maximal value and reached maximal value after the total perfusion peak. $C_{R B C}$ reached maximal value both before and after release and was at $91 \%$ of maximal value at time for perfusion peak on average. After occlusion, the return to baseline values was slower for $\mathrm{SO}_{2}$ than for $\mathrm{C}_{\mathrm{RBC}}$ and the perfusion estimates. This could possibly be caused by a rapid inflow of oxygenated blood at release where the oxygen supply exceeds the demand of the tissue. Even after blood flow has returned to baseline values, $\mathrm{SO}_{2}$ is higher than baseline. However, the detailed mechanism is still unknown.

Time to peak upon release after arterial occlusion was similar for conventional perfusion and total perfusion, and agrees with findings by Yvonne-Tee et al [29] for conventional perfusion on forearm. The inflation and deflation time of the cuff were similar in our study, since it could have an effect on time to peak [29]. The distance between the cuff and measurement site has also an effect on time to peak [30]. There was a difference in time to peak for the perfusion speed regions, where perfusion for speeds between $0-1 \mathrm{~mm} / \mathrm{s}$ has the shortest time to peak and, interestingly enough, perfusion for the middle speed region (1-10 $\mathrm{mm} / \mathrm{s}$ ) has the longest.

$\mathrm{SO}_{2}$ reached slightly below zero during the arterial occlusion for 10 out of 16 cases. This is an effect of the restrictions on the oxygen parameter, which allows for modeled oxygenation values both below 0 and above $100 \%$. By allowing for oxygenation values outside the physically correct range i.e. 0 to $100 \%$, the oxygen output parameter is allowed to fluctuate around the borders and therefore can attain a mean value of 0 or 100 . The criteria on the HemArea takes care of the cases were the amount of blood in the sampling area is too low to accurately estimate the oxygen saturation. $\mathrm{SO}_{2}$ from one subject was excluded during local heating and arterial occlusion, and from another subject during venous occlusion because of low HemArea. This could be an effect of the heterogeneity of the skin where a different site could produce a different estimate on the HemArea, or an individual difference in skin blood flow since the same subject was excluded from two provocations and also had the lowest $\mathrm{SO}_{2}$ among all subjects in the third. 
In conclusion, by integrating diffuse reflectance spectroscopy and laser Doppler flowmetry in a joint skin model, and by presenting oxygen saturation, RBC tissue fraction and speed resolved perfusion in absolute units, it is possible to achieve new insight into the microcirculatory system. Oxygen saturation, RBC tissue fraction and perfusion have different responses and different time to response during the three provocations which can be quantified by the method. With speed resolved perfusion it is possible to see the different responses for different vascular beds and with absolute units a comparison between individuals and sites can easier be obtained. This new optical method may provide further insight into the microcirculatory status and therefore also aid in the assessment of vascular dysfunction on a systemic level by using microcirculation as a model.

\section{Acknowledgement}

This study was financed by VINNOVA and Perimed $A B$ through the SamBIO research collaboration program between companies and academia within bioscience (VINNOVA D.no. 2008-00149) and the Research\&Grow program (VINNOVA D.no 2011-03074), and also by NovaMedTech supported by the European Union Regional Development Fund.

The authors would also like to thank PhD Sara Bergstrand for valuable discussions regarding the manuscript.

\section{References}

1. Debbabi, H., et al., Noninvasive assessment of endothelial function in the skin microcirculation. American Journal of Hypertension, 2010. 23(5): p. 541-546.

2. Ijzerman, R.G., et al., Individuals at increased coronary heart disease risk are characterized by an impaired microvascular function in skin. European Journal of Clinical Investigation, 2003. 33(7): p. 536-542.

3. Holowatz, L.A., C.S. Thompson-Torgerson, and W.L. Kenney, The human cutaneous circulation as a model of generalized microvascular function. Journal of Applied Physiology, 2008. 105(1): p. 370-372.

4. Verma, S., M.R. Buchanan, and T.J. Anderson, Endothelial Function Testing as a Biomarker of Vascular Disease. Circulation, 2003. 108(17): p. 2054-2059.

5. Blanco, M., et al., Platelets, inflammation, and atherothrombotic neurovascular disease: The role of endothelial dysfunction. Cerebrovascular Diseases, 2005. 20(SUPPL. 2): p. 32-39.

6. Yeboah, J., et al., Brachial flow-mediated dilation predicts incident cardiovascular events in older adults: The cardiovascular health study. Circulation, 2007. 115(18): p. 2390-2397.

7. Lindbergh, T., et al., Intramyocardial oxygen transport by quantitative diffuse reflectance spectroscopy in calves. Journal of biomedical optics, 2010. 15(2): p. 027009.

8. Lindbergh, T., et al., Improved model for myocardial diffuse reflectance spectra by including mitochondrial cytochrome aa3, methemoglobin, and inhomogenously distributed RBC. Journal of biophotonics, 2011. 4(4): p. 268-76.

9. Palmer, G.M. and N. Ramanujam, Monte Carlo-based inverse model for calculating tissue optical properties. Part I: Theory and validation on synthetic phantoms. Applied optics, 2006. 45(5): p. 1062-71.

10. Roustit, M. and J.L. Cracowski, Non-invasive Assessment of Skin Microvascular Function in Humans: An Insight Into Methods. Microcirculation, 2012. 19(1): p. 47-64.

11. Cracowski, J.L., et al., Methodological issues in the assessment of skin microvascular endothelial function in humans. Trends in Pharmacological Sciences, 2006. 27(9): p. 503-508.

12. Fredriksson, I., M. Larsson, and T. Strömberg, Model-based quantitative laser Doppler flowmetry in skin. Journal of biomedical optics, 2010. 15(5): p. 057002.

13. Fredriksson, I., et al., Inverse Monte Carlo in a multilayered tissue model: merging diffuse reflectance spectroscopy and laser Doppler flowmetry. J Biomed Opt, 2013. 18(12): p. 127004.

14. Ingemar, F., L. Marcus, and S. Tomas, Model-Based Quantification of Skin Microcirculatory Perfusion, in Computational Biophysics of the Skin. 2014, Pan Stanford Publishing. p. 395-418.

15. Liebert, A., N. Zołek, and R. Maniewski, Decomposition of a laser-Doppler spectrum for estimation of speed distribution of particles moving in an optically turbid medium: Monte Carlo validation study. Physics in Medicine and Biology, 2006. 51(22): p. 5737-5751.

16. Kuliga, K.Z., et al., Dynamics of Microvascular Blood Flow and Oxygenation Measured Simultaneously in Human Skin. Microcirculation, 2014. 21(6): p. 562-573. 
17. Beckert, S., et al., The impact of the micro-lightguide $\mathrm{O} 2 \mathrm{C}$ for the quantification of tissue ischemia in diabetic foot ulcers. Diabetes Care, 2004. 27(12): p. 2863-2867.

18. Fitzpatrick, T.B., The validity and practicality of sun-reactive skin types I through VI. Archives of Dermatology, 1988. 124(6): p. 869-871.

19. Fredriksson, I., M. Larsson, and T. Strömberg, Inverse Monte Carlo method in a multilayered tissue model for diffuse reflectance spectroscopy. Journal of Biomedical Optics, 2012. 17(4).

20. Fredriksson, I., et al. Improved calibration procedure for laser Doppler perfusion monitors. in Progress in Biomedical Optics and Imaging - Proceedings of SPIE. 2011.

21. Strömberg, T., et al., Microcirculation assessment using an individualized model for diffuse reflectance spectroscopy and conventional laser Doppler flowmetry. J Biomed Opt, 2014. 19(5): p. 57002.

22. Bland, M., An introduction to medical statistics. Oxford medical publications. 2000: Oxford : Oxford University Press, 2000

3. ed.

23. Roustit, M., et al., Reproducibility and methodological issues of skin post-occlusive and thermal hyperemia assessed by single-point laser Doppler flowmetry. Microvascular Research, 2010. 79(2): p. 102-108.

24. Bernjak, A., et al., Coherence between fluctuations in blood flow and oxygen saturation. Fluctuation and Noise Letters, 2012. 11(1).

25. Fredriksson, I., et al., Reduced arteriovenous shunting capacity after local heating and redistribution of baseline skin blood flow in type 2 diabetes assessed with velocity-resolved quantitative laser Doppler flowmetry. Diabetes, 2010. 59(7): p. 1578-84.

26. Fredriksson, I., M. Larsson, and T. Strömberg, Measurement depth and volume in laser Doppler flowmetry. Microvascular research, 2009. 78(1): p. 4-13.

27. Moppett, I., Basic principles of control of regional blood flow in vascular beds. Surgery (United Kingdom), 2012. 30(8): p. 365-369.

28. Pries, A.R., T.W. Secomb, and P. Gaehtgens, Biophysical aspects of blood flow in the microvasculature. Cardiovascular Research, 1996. 32(4): p. 654-667.

29. Yvonne-Tee, G.B., et al., Reproducibility of different laser Doppler fluximetry parameters of postocclusive reactive hyperemia in human forearm skin. Journal of Pharmacological and Toxicological Methods, 2005. 52(2): p. 286-292.

30. Roustit, M. and J.L. Cracowski, Assessment of endothelial and neurovascular function in human skin microcirculation. Trends in Pharmacological Sciences, 2013. 34(7): p. 373-384. 